\title{
SYNTHESIS AND IN-VITRO CYTOTOXIC ACTIVITY OF NOVEL BENZO[b]PHENAZINE-6,11-DIONE AND 1,4- NAPHTHOQUINONE DERIVATIVES
}

Maha M. A. Khalifa ${ }^{1 *}$, Magda M. F. Ismail ${ }^{1}$ and Eman Noaman ${ }^{2}$

${ }^{1}$ Department of Pharmaceutical Chemistry, Faculty of Pharmacy (Girls), Al-Azhar University, Nasr City, Cairo, Egypt

${ }^{2}$ Department of Radiation Biology, Natural Center for Radiation Research and Technology, Atomic Energy Authority, Cairo, Egypt

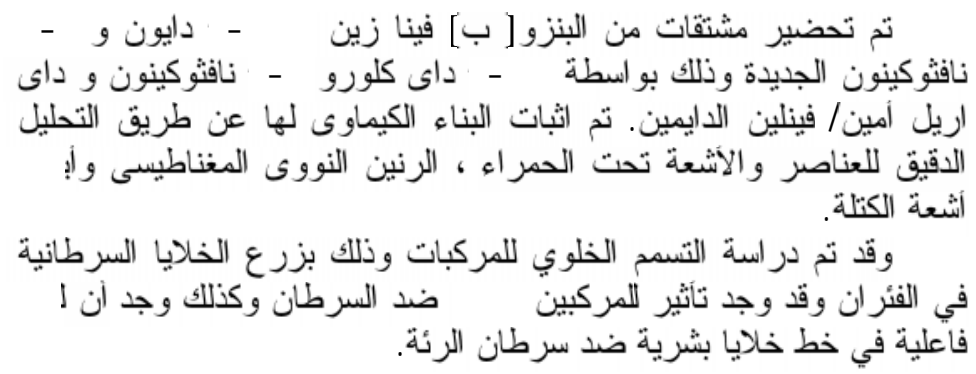

5,12-Dihydrobenzophenazine-6,11-diones, 2-Arylamino-3chloro-1,4-naphthoquinones and 6,11-dihydrobenzo[b]phenazine6,11-diones, were synthesized from 2,3-dichloro-1,4-naphthoquinone and arylamines/phenylenediamines. Studying the cytotoxicity using EAC and human cell lines revealed that 5,12dihydrobenzo[b]phenazine-6,11-dione (3) and 3-chloro-2-(2pyridylamino)-1,4-naphthoquinone (10) showed selective cytotoxicity against the human lung carcinoma cell line (H460) superior to doxorubicin. Compound 3 (16.25 uM) was 1.3 times higher than that of doxorubicin. However, IC50 value of compound 10 was $9.90 u M$ which was 2 times higher than that (20.10 uM) of doxorubicin. These compounds were inactive against liver carcinoma (HEPG2), brain tumor (U251), cervix carcinoma (HELA) and breast carcinoma (MCF7) cell lines.

Received in 17/3/2008, Received in revised form in 17/6/2008 \& Accepted in 19/6/2008

${ }^{*}$ Corresponding auther e-mail address: maha_khalifa@hotmail.com. 


\section{INTRODUCTION}

Studies on the activity of heterocyclic quinones containing nitrogen showed that the number and position of nitrogens are considerably important for cytotoxicity $^{1}$. The diazanapthoquinone were proved to be the most active compounds in comparison with napthoquinone and quinolidinedione $^{2}$. Another structural requirements for the antitumor activity is the p-quinone moiety in the non heterocyclic ring, however o-quinone gave decreased activity $^{3 \& 4}$. One of the proposed mechanisms of coplanar polycyclic compounds is that they act as topoisomerase inhibitor via DNA intercalation $^{5-7}$. The topoisomerase are essential enzymes in the regulation of DNA topology which is required if cells are to divide and proliferate ${ }^{8}$. The phenazines have been shown to fulfill the fundamental physicochemical requirements for DNA intercalation ${ }^{9}$. However it was reported by Johnson and approved by Lee ${ }^{10}$ that the antitumor activity is enhanced as more heterocyclic rings were annulated to the heteroquinone ring as well as it's dependency on the number of nitrogen atoms. Most of the previously reported phenazines contain unsaturated nitrogen atom $^{9 \& 10}$, however in the present investigation, a novel series of substituted 5,12-dihydrobenzo- [b]phenazine-6,11-diones and their bioisosteres ${ }^{11 \& 12}$ having planar ring and $p$-conjugated ketone groups containing a nitrogen atom which may enable additional hydrogen bonding with DNA was synthesized.

Prompted by the fact that streptonigrin which is an alkaloid having excellent antitumor activities ${ }^{13}$. We also synthesized 2Arylamino-3-chloro-1,4-naphthoquinones and their corresponding rigid structures. All the compounds were then submitted for preliminary in vitro screening for cytotoxic activity.

\section{EXPERIMENTAL}

\section{Chemistry}

Melting points were determined on electrothermal 9100 digital melting point apparatus and were uncorrected. ${ }^{1} \mathrm{HNMR}$ spectra were recorded in DMSO- $\mathrm{d}_{6}$ on Varian Gemini 200 (200 MHz) using tetramethylsilane (TMS) as an internal standard (chemical shift in $\delta$ ppm). The IR spectra were performed on a Perckin-Elmer 1600 FTIR in $\mathrm{KBr}$ pellets. Elemental microanalysis $(\mathrm{C}, \mathrm{H}, \mathrm{N})$ were performed on a Perkin-Elmer 2400 analyzer from vacuum-dried samples at the micro analytical unit of Cairo University. All compounds were within $\pm 0.4 \%$ of the theoretical values. The mass spectra were recorded on a HewlettPackard 5988-A instrument at 70 
$\mathrm{eV}$. Chemicals were purchased from E. Merck (Darmstadt, Germany), Sigma-Aldrich (Germany); solvents used were of the highest grade.

General procedure for the preparation of 5,12-dihydrosubstitutedbenzo $[b]$ phenazine- 6 , 11-diones (3-6)

o-Phenylene diamine derivatives,

$2(0.5 \mathrm{mmol})$ was added to a solution of 2,3-dichloro-1,4-napthoquinone $1(0.5 \mathrm{mmol})$ in DMF (20 $\mathrm{mL}$ ) and heated under reflux for 5 h. The reaction mixture was allowed to cool, poured into icewater and the product obtained was filtered, dried and recrystallized from appropriate solvent (Table 1).

\section{5,12-Dihydrobenzo[ $b]$ phenazine-}

6,11-dione (3). IR $\left(\mathrm{KBr}, \mathrm{cm}^{-1}\right)$ : 3423 (NH), 1676 (CO), ${ }^{1}$ HNMR (DMSO- $\left.d_{6}\right): \delta$ 6.90-7.33 $(\mathrm{m}, 4 \mathrm{H}$, $\mathrm{H}_{1}, \mathrm{H}_{2}, \mathrm{H}_{3}, \mathrm{H}_{4}$ ), 7.47-8.16 (m, 4H, napthoquinone $-\mathrm{Hs}), 9.20 \quad(\mathrm{~s}, 2 \mathrm{H}$, $2 \mathrm{NH}) . \mathrm{MS}(\mathrm{m} / \mathrm{z} \%): 280\left(\mathrm{M}+\mathrm{H}_{2} \mathrm{O}\right.$, 100), 252 (24.9), 217 (29.2), 188 (11.4).

2-Methyl-5,12-dihydrobenzo[b]phenazine-6,11-dione (4). IR ( $\mathrm{KBr}$, $\left.\mathrm{cm}^{-1}\right): 3270(\mathrm{NH}), 1666$ (CO), ${ }^{1} \mathrm{HNMR}$ (DMSO- $\left.d_{6}\right): \delta 2.64(\mathrm{~s}, 3 \mathrm{H}$, $\left.\mathrm{CH}_{3}\right), 7.27\left(\mathrm{~s}, 1 \mathrm{H}, \mathrm{H}_{1}\right), 7.79-7.93$ (m, $2 \mathrm{H}, \mathrm{H}_{3}, \mathrm{H}_{4}$ ), 8.06-8.21 (m, 4H, napthoquinone $-\mathrm{Hs}), 9.20(\mathrm{~s}, 1 \mathrm{H}$, $\mathrm{NH}), 9.28(\mathrm{~s}, 1 \mathrm{H}, \mathrm{NH}) . \mathrm{MS}(\mathrm{m} / \mathrm{z}$ \%): $294\left(\mathrm{M}+\mathrm{H}_{2} \mathrm{O}, 100\right), 229$ (29.8), 147 (17.5), 102 (17.5).
2,3-Dimethyl-5,12-dihydrobenzo[b]phenazine-6,11-dione (5). IR $\left(\mathrm{KBr}, \mathrm{cm}^{-1}\right): 3244(\mathrm{NH}), 1654(\mathrm{CO})$, ${ }^{1} \mathrm{HNMR}\left(\mathrm{DMSO}-d_{6}\right): \delta 2.08(\mathrm{~s}, 6 \mathrm{H}$, $\left.2 \mathrm{CH}_{3}\right), 6.26\left(\mathrm{~s}, 2 \mathrm{H}, \mathrm{H}_{1}, \mathrm{H}_{4}\right), 7.28$ $8.40(\mathrm{~m}, 4 \mathrm{H}$, naphthoquinone- $\mathrm{Hs})$, 10.66 (s, 2H, $2 \mathrm{NH}$ ).

2-Chloro-5, 12-dihydrobenzo[b]phenazine-6,11-dione (6). IR ( $\mathrm{KBr}$, $\left.\mathrm{cm}^{-1}\right): 3320(\mathrm{NH}), 1672 \quad(\mathrm{CO})$, ${ }^{1} \mathrm{HNMR}$ (DMSO- $d_{6}$ ): $\delta$ 7.89-7.96 (m, 3H, $\left.\mathrm{H}_{1}, \mathrm{H}_{3}, \mathrm{H}_{4}\right), 8.05-8.12$ (m, 4H,naphthoquinone-Hs), 9.02 (s, $2 \mathrm{H}, 2 \mathrm{NH})$

\section{2-Chloro-12H-benzo[b]phenox- azine-6,11-dione (7)}

To a solution of 2,3-dichloro1,4-napthoquinone $1(0.5 \mathrm{mmol})$ in EtOH $(30 \mathrm{~mL})$, add $\mathrm{KOH}(0.39 \mathrm{~g}$, $0.01 \mathrm{~mol})$ and 4-chloro-o-aminophenol $(0.5 \mathrm{mmol})$ Then the mixture was heated under reflux for $6 \mathrm{~h}$, allowed to cool, poured into icewater and the product obtained was filtered, dried and recrystallized from appropriate solvent (Table 1). 7: $\mathrm{IR}\left(\mathrm{KBr}, \mathrm{cm}^{-1}\right): 3180(\mathrm{NH}), 1640$ (CO), ${ }^{1} \mathrm{HNMR}$ (DMSO- $\left.d_{6}\right): \delta 6.53$ $6.65\left(\mathrm{~m}, 2 \mathrm{H}, \mathrm{H}_{3}, \mathrm{H}_{4}\right), 7.65-7.88(\mathrm{~m}$, $\left.2 \mathrm{H}, \mathrm{H}_{8}, \mathrm{H}_{9}\right), 8.01-8.06\left(\mathrm{~m}, 3 \mathrm{H}, \mathrm{H}_{1}\right.$, $\left.\mathrm{H}_{7}, \mathrm{H}_{10}\right), 9.14\left(\mathrm{~s}, 1 \mathrm{H}, \mathrm{NH}, \mathrm{D}_{2} \mathrm{O}\right.$ exchangeable). MS (m/z \%): 299 $(\mathrm{M}+2,30.1), 297\left(\mathrm{M}^{+}, 50.4\right), 281$ (85.8), 253 (51.3), 220 (56.6), 50 (100). 
Table 1: Physical properties and molecular formula of the synthesized compounds.

\begin{tabular}{|c|c|c|c|c|c|c|}
\hline $\begin{array}{l}\text { Compd } \\
\text { No. }\end{array}$ & $\begin{array}{l}\text { M.P. } \\
\left({ }^{\circ} \mathrm{C}\right)\end{array}$ & Solvent of Cryst. & $\begin{array}{l}\text { Yield } \\
(\%)\end{array}$ & $\begin{array}{l}\text { Mol.Formula } \\
\text { M.Wt }\end{array}$ & Calcd. & Found \\
\hline 3 & $198-200$ & $\mathrm{EtOH}$ & 90 & $\begin{array}{c}\mathrm{C}_{16} \mathrm{H}_{10} \mathrm{~N}_{2} \mathrm{O}_{2} \mathrm{H}_{2} \mathrm{O} \\
280.26\end{array}$ & $\begin{array}{l}\text { C } 68.57 \\
\text { H } 4.28 \\
\text { N } 9.99\end{array}$ & $\begin{array}{l}68.99 \\
3.85 \\
9.99\end{array}$ \\
\hline 4 & $266-68$ & EtOH & 90 & $\begin{array}{c}\mathrm{C}_{17} \mathrm{H}_{12} \mathrm{~N}_{2} \mathrm{O}_{2} \cdot \mathrm{H}_{2} \mathrm{O} \\
294.29\end{array}$ & $\begin{array}{l}\text { C } 69.31 \\
\text { H } 4.75 \\
\text { N } 9.51\end{array}$ & $\begin{array}{l}69.72 \\
4.56 \\
9.91\end{array}$ \\
\hline 5 & $246-48$ & Pet ether/ $\mathrm{CH}_{2} \mathrm{Cl}_{2}$ & 80 & $\begin{array}{c}\mathrm{C}_{18} \mathrm{H}_{14} \mathrm{~N}_{2} \mathrm{O}_{2 .} .0 .5 \mathrm{H}_{2} \mathrm{O} \\
299.32\end{array}$ & $\begin{array}{l}\text { C } 72.16 \\
\text { H } 5.01 \\
\text { N } 9.35\end{array}$ & $\begin{array}{l}72.10 \\
4.90 \\
9.35\end{array}$ \\
\hline 6 & $255-57$ & $\mathrm{EtOH}$ & 50 & $\begin{array}{c}\mathrm{C}_{16} \mathrm{H}_{9} \mathrm{ClN}_{2} \mathrm{O}_{2} \\
296.71\end{array}$ & $\begin{array}{l}\text { C } 64.77 \\
\text { H } 3.06 \\
\text { N } 9.43\end{array}$ & $\begin{array}{l}64.50 \\
3.20 \\
9.26 \\
\end{array}$ \\
\hline 7 & $270-72$ & $\mathrm{EtOH}$ & 40 & $\begin{array}{c}\mathrm{C}_{16} \mathrm{H}_{8} \mathrm{ClNO}_{3} \\
297.69\end{array}$ & $\begin{array}{l}\text { C } 64.55 \\
\text { H } 2.71 \\
\text { N } 4.71\end{array}$ & $\begin{array}{l}64.80 \\
2.90 \\
4.95\end{array}$ \\
\hline 8 & $270-72$ & EtOH/Ether & 40 & $\begin{array}{c}\mathrm{C}_{16} \mathrm{H}_{9} \mathrm{NO}_{2} \mathrm{~S} \\
279.31\end{array}$ & $\begin{array}{l}\text { C } 68.80 \\
\text { H } 3.25 \\
\text { N } 5.01\end{array}$ & $\begin{array}{l}68.99 \\
3.20 \\
5.57 \\
\end{array}$ \\
\hline 10 & $143-46$ & $\mathrm{EtOH}$ & 80 & $\begin{array}{c}\mathrm{C}_{15} \mathrm{H}_{9} \mathrm{ClN}_{2} \mathrm{O}_{2} \cdot 0.5 \mathrm{H}_{2} \mathrm{O} \\
293.70\end{array}$ & $\begin{array}{l}\text { C } 61.22 \\
\text { H } 3.74 \\
\text { N } 9.53\end{array}$ & $\begin{array}{l}61.25 \\
3.93 \\
9.45\end{array}$ \\
\hline 11 & $193-95$ & EtOH/Ether & 65 & $\begin{array}{c}\mathrm{C}_{18} \mathrm{H}_{14} \mathrm{ClNO}_{2} .0 .25 \mathrm{H}_{2} \mathrm{O} \\
316.26\end{array}$ & $\begin{array}{l}\text { C } 68.29 \\
\text { H } 4.58 \\
\text { N } 4.42\end{array}$ & $\begin{array}{l}68.63 \\
4.43 \\
4.37 \\
\end{array}$ \\
\hline 12 & $286-88$ & $\mathrm{EtOH}$ & 30 & $\begin{array}{c}\mathrm{C}_{15} \mathrm{H}_{7} \mathrm{~N}_{3} \mathrm{O}_{2} \\
261.20\end{array}$ & $\begin{array}{l}\text { C } 68.96 \\
\text { H } 2.70 \\
\text { N } 16.07\end{array}$ & $\begin{array}{l}68.68 \\
3.01 \\
16.55\end{array}$ \\
\hline 13 & $186-88$ & EtOH/Ether & 42 & $\begin{array}{c}\mathrm{C}_{18} \mathrm{H}_{12} \mathrm{~N}_{2} \mathrm{O}_{2} \\
288.30\end{array}$ & $\begin{array}{l}\text { C } 74.99 \\
\text { H } 4.20 \\
\text { N } 9.71\end{array}$ & $\begin{array}{l}75.17 \\
4.14 \\
9.79\end{array}$ \\
\hline
\end{tabular}


2-Chloro-12H-benzo[b]phenothiazine-6,11-dione (8)

The above procedure was followed using 4-chloro-o-aminothiophenol $(0.5 \mathrm{mmol})$ and the product obtained was purified using column chromatography using cyclohexane : EtOAc (1:3) as an eluant. IR $\left(\mathrm{KBr}, \mathrm{cm}^{-1}\right): 3190(\mathrm{NH})$, $1640(\mathrm{CO}),{ }^{1} \mathrm{HNMR}$ (DMSO- $d_{6}$ ): $\delta$ $7.4(\mathrm{t}, 1 \mathrm{H}, \mathrm{H}), 7.6\left(\mathrm{t}, 1 \mathrm{H}, \mathrm{H}_{3}\right), 7.69-$ $7.80\left(\mathrm{~m}, 2 \mathrm{H}, \mathrm{H}_{1}, \mathrm{H}_{4}\right), 7.85-7.90(\mathrm{t}$, $\left.2 \mathrm{H}, \mathrm{H}_{8}, \mathrm{H}_{9}\right), 8.10$ (d, $2 \mathrm{H}, \mathrm{H}_{7}, \mathrm{H}_{10}$ ), $9.20(\mathrm{~s}, 1 \mathrm{H}, \mathrm{NH})$.

General procedure for the preparation of 2-arylamino-3chloro-1,4-naphthoquinones (10 and 11)

2-aminopyridine/2-ethylaniline $(0.5 \mathrm{mmol})$ was added to a solution of 2,3-dichloro-1,4-napthoquinone $\mathbf{1}$ $(1.135 \mathrm{~g}, 0.5 \mathrm{mmol})$ in ethanol (30 $\mathrm{mL}$ ) and heated under reflux for 3 $\mathrm{h}$. The reaction mixture was cooled and then filtered. The product was crystallized from the appropriate solvent (Table 1).

3-chloro-2-(2-pyridylamino)-1,4napthoquinone $(\mathbf{1 0})$. IR $(\mathrm{KBr}$, $\left.\mathrm{cm}^{-1}\right): 3327(\mathrm{NH}), 1678$ (CO), 1584 $(\mathrm{C}=\mathrm{N}) .{ }^{1} \mathrm{H}$ NMR (DMSO- $\left.d_{6}\right): \delta$ 7.89-7.96 (m, 4H, H $\mathrm{H}_{3}, \mathrm{H}_{4}, \mathrm{H}_{5}, \mathrm{H}_{6}$ ), 8.06-8.09 (m, 4H, napthoquinone$\mathrm{Hs}), \quad 9.20 \quad\left(\mathrm{~s}, \quad 1 \mathrm{H}, \quad \mathrm{NH}, \quad \mathrm{D}_{2} \mathrm{O}\right.$ exchangeable).

3-Chloro-2-(2-ethylphenylamino)1,4-napthoquinone (11). IR ( $\mathrm{KBr}$, $\left.\mathrm{cm}^{-1}\right)$ : $3328(\mathrm{NH}), 1678$ (CO);
${ }^{1} \mathrm{H}$ NMR (DMSO- $\left.d_{6}\right): \delta 1.13(\mathrm{t}, 3 \mathrm{H}$, $\mathrm{CH}_{3}, J=7.5 \mathrm{~Hz}$ ), 2.63 (q, $2 \mathrm{H}, \mathrm{CH}_{2}$, $J=7.5 \mathrm{~Hz}), 7.14-7.25\left(\mathrm{~m}, 4 \mathrm{H}, \mathrm{H}_{3}\right.$, $\left.\mathrm{H}_{4}, \mathrm{H}_{5}, \mathrm{H}_{6}\right), 7.78-8.08\left(\mathrm{~m}, 2 \mathrm{H}, \mathrm{H}_{6}\right.$, $\left.\mathrm{H}_{7}\right), 8.09-8.11\left(\mathrm{~m}, 2 \mathrm{H}, \mathrm{H}_{5}, \mathrm{H}_{8}\right), 9.01$ (s, 1H, NH).

General procedure for the preparation of $1,5,12$-Triazanapthacene-6,11-dione and 6,11Dihydro-1-ethylbenzo $[b]$ phenazine-6,11-dione (12 and 13)

A mixture of $0.5 \mathrm{mmol}$ of $\mathbf{1 0}$ or 11 in $50 \mathrm{~mL}$ of DMF and $(0.65 \mathrm{~g}$, $0.01 \mathrm{~mol}$ ) of sodium azide, suspended in a little amount of water $(1 \mathrm{~mL})$ was heated on the steam bath overnight. The reaction was chilled, the filtered precipitate was extracted with methylene chloride and concentrated, and then the residue was purified by crystallization (Table 1).

1,5,12-Triaza-naphthacene-6,11dione (12). IR $\left(\mathrm{KBr}, \mathrm{cm}^{-1}\right): 1626$ $(\mathrm{C}=\mathrm{N}), \quad 1687 \quad(\mathrm{CO}) . \quad{ }^{1} \mathrm{H} \quad \mathrm{NMR}$ $\left(\right.$ DMSO- $\left.d_{6}\right): \delta 7.39\left(\mathrm{t}, 1 \mathrm{H}, \mathrm{H}_{3}\right), 7.59$ (d, $\left.1 \mathrm{H}, \mathrm{H}_{4}\right), 7.81\left(\mathrm{t}, 2 \mathrm{H}, \mathrm{H}_{8}, \mathrm{H}_{9}\right)$, 7.97-7.99 (m, 2H, $\left.\mathrm{H}_{7}, \mathrm{H}_{10}\right), 8.13$ (d, $\left.1 \mathrm{H}, \mathrm{H}_{2}\right)$.

\section{6,11-Dihydro-1-ethylbenzo[b]-} phenazine-6,11-dione (13). IR $\left(\mathrm{KBr}, \mathrm{cm}^{-1}\right): 1585 \quad(\mathrm{C}=\mathrm{N}), 1677$ (CO). ${ }^{1} \mathrm{H}$ NMR (DMSO- $d_{6}$ ): $\delta 1.38$ (t, $\left.3 \mathrm{H}, \mathrm{CH}_{3}, J=7.5 \mathrm{~Hz}\right), 3.35$ (q, $2 \mathrm{H}$, $\left.\mathrm{CH}_{2}, J=7.5 \mathrm{~Hz}\right), 7.95-8.06(\mathrm{~m}, 4 \mathrm{H}$, $\left.\mathrm{H}_{2}, \mathrm{H}_{3}, \mathrm{H}_{8}, \mathrm{H}_{9}\right), 8.20\left(\mathrm{~d}, 1 \mathrm{H}, \mathrm{H}_{4}\right)$, 8.30-8.33 (m, $2 \mathrm{H}, \mathrm{H}_{7}, \mathrm{H}_{10}$ ). MS (m/z \%): $288\left(\mathrm{M}^{+}, 17.0\right), 248$ (58.2), 232 (23.5), 221 (47.1). 
Methods of antitumor screening Activity against EAC experimental cell line

Animals, chemicals and facilities: Female Swiss albino mice weighing 25-30 g obtained from (the holding company of biological products and vaccines, VACSERA, Cairo, Egypt) were housed at a constant temperature $\left(24 \pm 2^{\circ} \mathrm{C}\right)$ with alternating $12 \mathrm{~h}$ light and dark cycles and fed standard laboratory food (Milad Co., Cairo Egypt) and water adlibitum. All chemicals and reagents were from Sigma-Aldrich Germany and Merck- Germany.

Ehrlich Ascites Carcinoma cells (EAC) were obtained by needle aspiration of ascetic fluid from preinoculated mice; under aseptic conditions. Tumor cells suspension $\left(2.5 \times 10^{6}\right.$ per $\left.\mathrm{mL}\right)$ was prepared. Tested compounds were prepared with various dilutions in DMSO (1 $\mathrm{mL})$. In a set of sterile test tubes 0.8 $\mathrm{mL}$ MBIR-1640, $0.1 \mathrm{~mL}$ of each of the tested compounds (corresponding to $0.34,0.265,0.177,0.088$ and $0.035 \mu \mathrm{M} / \mathrm{mL}$ ) and $0.8 \mathrm{~mL}$ of media (RBMI-contain glutamine and fetal calf serum as nutrient beside penicillin and streptomycin as antibiotics) were mixed then, 0.1 $\mathrm{mL}$ of tumor cell suspension was added. The test tubes were incubated at $37^{\circ} \mathrm{C}$ for $2 \mathrm{~h}$. Then, trypan blue exclusion test ${ }^{14}$ was carried out to calculate the percentage of non-viable cells.

\section{Activity against human cell lines}

Cells were plated in 96multiwell plate $\left(10^{4}\right.$ cells/well $)$ for $24 \mathrm{~h}$ before treatment with the compounds to allow attachment of cell to the wall of the plate. Different concentrations of the compounds under test $(0-36.00$ $\mu \mathrm{M} / \mathrm{mL}$ ) were solubilised in dimethyl-sulfoxide (DMSO) and were added to the cell monolayer of the five human cell lines. Monolayer cells were incubated with the compounds for $48 \mathrm{~h}$ at $37^{\circ} \mathrm{C}$ and in atmosphere of $5 \% \mathrm{CO}_{2}$. After 48 h, cells were fixed, washed and stained with sulforhodamine B stain. The color intensity was measured in an ELISA reader ${ }^{15}$. The relation between surviving fraction and drug concentration is plotted to get the survival curve for the active compounds. Statistical Analysis Student's $t$ test was used for analysis of the biochemical parameters. The data were expressed as mean \pm standard error $^{16}$.

\section{RESULTS AND DISCUSSION}

\section{Chemistry}

The preparation of target compounds was conducted according to the sequence of reactions are depicted in Schemes 1 and 2. Treatment of $\mathbf{1}$ with 1,2phenylenediamines, $\mathbf{2}$ in DMF under reflux condition ${ }^{2}$ afforded the desired compounds, 3-6. In analogy, 
<smiles>O=C1C(Cl)=C(Cl)C(=O)c2ccccc21</smiles>

1<smiles>[X]c1cc([X])c(N)cc1[X]</smiles><smiles>[Z]c1cc2cc([X])c3c(=O)c4ccccc4c(=O)c=3[nH]c2cc1[X]</smiles>

$\begin{array}{cccc}\text { Compd No } & \mathrm{X} & \mathrm{Y} & \mathrm{Z} \\ 3 & \mathrm{NH} & \mathrm{H} & \mathrm{H} \\ 4 & \mathrm{NH} & \mathrm{CH}_{3} & \mathrm{H} \\ 5 & \mathrm{NH} & \mathrm{CH}_{3} & \mathrm{CH}_{3} \\ 6 & \mathrm{NH} & \mathrm{Cl} & \mathrm{H} \\ 7 & \mathrm{O} & \mathrm{Cl} & \mathrm{H} \\ 8 & \mathrm{~S} & \mathrm{H} & \mathrm{H}\end{array}$

Scheme 1: Synthesis of compounds 3-8.

Reaction conditions: $\mathrm{i}=\mathrm{DMF} /$ reflux 5 h, 7,8: ii $=\mathrm{KOH} / \mathrm{ETOH} /$ reflux 6 h.<smiles>O=C1C(Cl)=C(Cl)C(=O)c2ccccc21</smiles><smiles>[Y]c1ccccc1N</smiles>

9

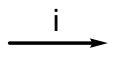

$12 ; \mathrm{X}=\mathrm{N}, \mathrm{Y}=-$

$13 ; \mathrm{X}=\mathrm{C}, \mathrm{Y}=\mathrm{C}_{2} \mathrm{H}_{5}$<smiles>[Y]c1ccccc1NC1=C(Cl)C(=O)c2ccccc2C1=O</smiles>

$11 ; \mathrm{X}=\mathrm{C}, \mathrm{Y}=\mathrm{C}_{2} \mathrm{H}_{5}$

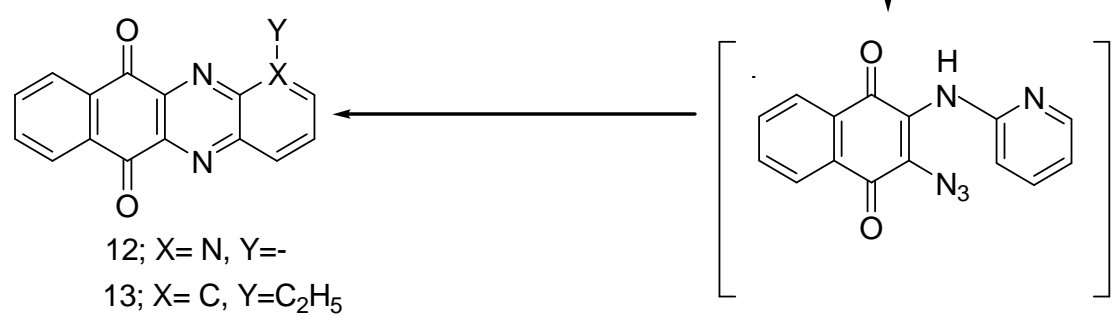

Scheme 2: Synthesis of compounds 10-14.

Reaction conditions: $\mathrm{i}=$ Ethanol/reflux $3 \mathrm{~h}, \mathrm{ii}=\mathrm{NaN}_{3} / \mathrm{DMF} /$ reflux $24 \mathrm{~h}$. 
reaction of 2-amino-4-chlorophenol or thiophenol with 1 using $\mathrm{KOH}$ in refluxing ethanol for furnished the desired bioisosteres, $\mathbf{7}$ and $\mathbf{8}$.

2-Arylamino-3-chloro-1,4-naphthoquinones, 10, 11 were obtained by reacting 2,3-dichloro-1,4napthoquinone, 1 with arylamines 9 in ethanol. The latter were reacted with sodium azide in DMF at 90$100^{\circ} \mathrm{C}$ overnight ${ }^{10}$ to give our target compounds, 1,5,12-Triaza-napthacene-6,11-dione and 6,11-dihydro1-ethylbenzo[ $b]$ phenazine- 6,11 -

dione respectively $(\mathbf{1 2}, \mathbf{1 3})$. The reaction is believed to proceed via the formation of the unstable intermediate, the 2-azido derivative $^{10}$.

\section{Cytotoxic activity}

Activities against Ehrlich Ascites

\section{Carcinoma (EAC) cell line}

These biological studies were performed at the National Center for Radiation Research and Technology (NCRRT), Cairo, Egypt. A preliminary screening on the new compounds was performed against EAC cells ${ }^{17}$. The tumor cell suspensions were incubated with different concentrations in $\mathrm{mM} / \mathrm{mL}^{15 \& 18}$ (Table 2).

In vitro antitumor screening of 5,12-dihydrobenzo[ $b]$ phenazine6,11-diones (3-6) and their classic bioisosteres $(\mathbf{7}, \mathbf{8})$ revealed that, the unsubstituted derivative, $\mathbf{3}$ exhibited higher cytotoxic activity (IC50 $=0.035 \mathrm{mM})$ compared to that of the standard. The presence of electron-donating substituent $\left(\mathrm{CH}_{3}\right)$ at position 2 , decreased the activity $(\mathbf{4}$, IC50 $=>0.354 \mathrm{mM})$. Introduction of another $\mathrm{CH}_{3}$ group at position 3 in compound 5; (IC50 $=>0.354 \mathrm{mM})$ showed also decrease in cytotoxic activity. On the other hand, introduction of electron-withdrawing group $(\mathrm{Cl})$ at position 2 of benzo[ $b]$ phenazine nucleus compound $\mathbf{6}$ enhanced its cytotoxicity $($ IC50 $=>0.035 \mathrm{mM})$ which may enable nitrogen at position 5 of hydrogen bonding with DNA.

Classic bioisosteres $(\mathbf{7}, \mathbf{8})$, in which the $\mathrm{NH}$ group at position 5 is replaced by $\mathrm{O}$ or $\mathrm{S}$ respectively, showed great reduction in the cytotoxic activity, IC50 $=0.088$, $0.354 \mathrm{mM}$ respectively.

3-Chloro-2-(2-pyridylamino)1,4-naphthoquinone (10) was the most cytotoxic compound (IC50 = $<0.035 \mathrm{mM}$ ) which is higher than that of the standard, doxorubicin $($ IC50 $=0.15 \mathrm{mM})$. However, rigidification of this compound (12) abolished the antitumor activity. The active compounds $(\mathbf{3}, \mathbf{1 0})$ were further tested at lower concentrations $\quad(0-36.00 \mu \mathrm{M} / \mathrm{mL})$. The relationship between surviving fraction and drug concentration was plotted to obtain the survival curve of EAC cell line. The response parameter calculated was IC50 value which corresponds to the compound concentration causing $50 \%$ mortality in net cells (Table 2 , Fig. 1). 
Table 2: In-vitro cytotoxic activity of some selected synthesized compounds.

\begin{tabular}{||c|c|c|c|c|c|c||}
\hline \multirow{2}{*}{$\begin{array}{c}\text { Compd. } \\
\text { No. }\end{array}$} & \multicolumn{5}{|c|}{ Non Viable cells (\%) } & \multirow{2}{*}{$\begin{array}{c}\text { IC }_{50} \\
(\mathrm{mM} / \mathrm{mL})\end{array}$} \\
\cline { 2 - 6 } & 0.035 & 0.088 & 0.177 & 0.265 & 0.354 & \\
\cline { 2 - 6 } $\begin{array}{c}\text { Article I } \\
\text { doxorubicin }\end{array}$ & $12 \pm 1.01$ & $20 \pm 1.30$ & $65 \pm 2.34$ & $75 \pm 4.11$ & $95 \pm 2.11$ & 0.15 \\
\hline 3 & $50 \pm 1.2-$ & $70 \pm 1.9$ & $90 \pm 2.9$ & $95 \pm 3.1$ & $100 \pm 1.0$ & 0.035 \\
\hline 4 & $-^{\mathrm{a}}$ & $-^{\mathrm{a}}$ & $-^{\mathrm{a}}$ & 10 & 20 & $>0.354$ \\
\hline 5 & $-^{\mathrm{a}}$ & $2 \pm$ & $5 \pm$ & $10 \pm$ & $10 \pm$ & $>0.354$ \\
\hline 6 & $55 \pm 3.5$ & $60 \pm 3.2$ & $67 \pm 4.1$ & $75 \pm 2.5$ & $84 \pm 2.1$ & $<0.035$ \\
\hline 7 & $40 \pm 1.4$ & $50 \pm 2.1$ & $70 \pm 2.3$ & $77 \pm 3.0$ & $100 \pm 1.01$ & 0.088 \\
\hline 8 & $-^{\mathrm{a}}$ & $-{ }^{\mathrm{a}}$ & 2 & 5 & 5 & $>0.354$ \\
\hline $\begin{array}{c}\text { Article II } \\
10\end{array}$ & $89 \pm 4.8$ & $90 \pm 4.3$ & $95 \pm 2.1$ & $95 \pm 2.9$ & $100 \pm 1.0$ & $<0.035$ \\
\hline 11 & $30 \pm 2.2$ & $11 \pm 1.3$ & $30 \pm 3.2$ & $44 \pm 3.4$ & $50 \pm 4.1$ & 0.354 \\
\hline 12 & $-^{\mathrm{a}}$ & $-^{\mathrm{a}}$ & $-^{\mathrm{a}}$ & $-^{\mathrm{a}}$ & $-^{\mathrm{a}}$ & - \\
\hline 13 & $3 \pm 0.01$ & $4 \pm 0.011$ & $7 \pm 0.012$ & $11.7 \pm 0.011$ & $18 \pm 0.09$ & $>0.354$ \\
\hline
\end{tabular}

${ }^{\text {a }}$ All cells are alive.

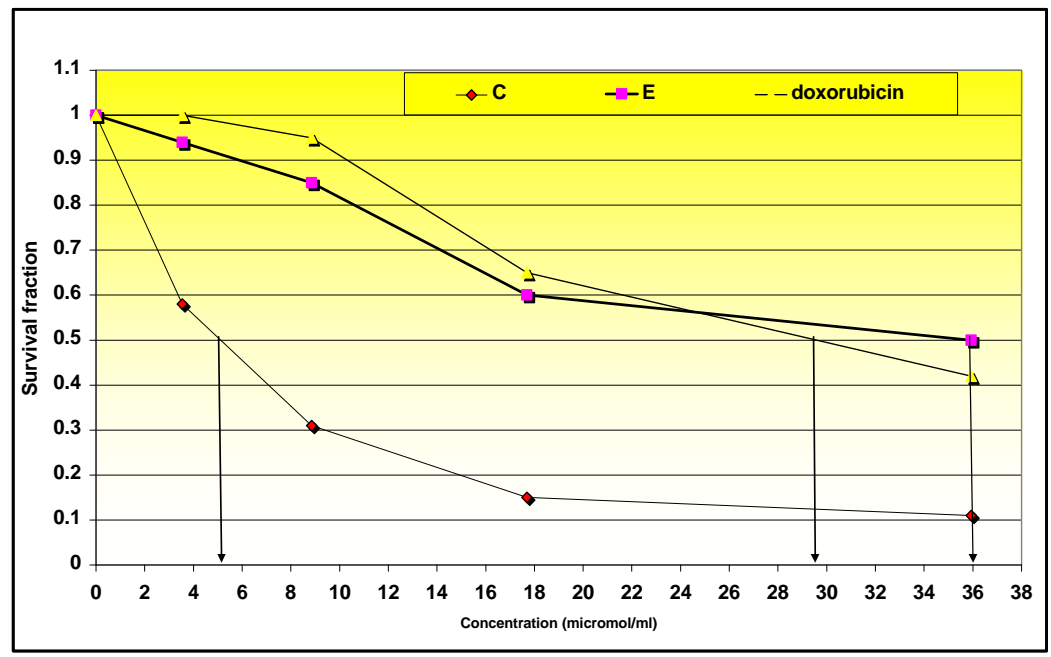

E: compound No. 3; C: compound No. 10.

Fig. 1: Cytotoxic activity of compounds 3, 10 and doxorubicin on cell survival of Ehrlich Ascites Carcinoma cell line (EAC) arrows represented IC50 concentration. 


\section{Activity against human cell lines}

Antitumor screening was performed at the National Cancer Institute, Cancer Biology Department, and Cairo, Egypt. Potential cytotoxicity of the active compounds $\mathbf{3}$ and $\mathbf{1 0}$ was tested using the method of Skehan et al Five human cell lines: liver carcinoma (HEPG2), brain tumor (U251), cervix carcinoma (HELA), breast carcinoma (MCF7) and lung carcinoma (H450) were incubated with five concentrations (0-36.00) $\mu \mathrm{M} / \mathrm{ml}^{15 \& 18}$ for each compound 5,12-dihydrobenzo[ $b]$ phenazine-

6,11-dione (3) and 3-Chloro-2-(2pyridylamino)-1,4-naphthoquinone
(10) showed selective cytotoxicity against the human lung carcinoma cell line $(\mathrm{H} 460)$ superior to doxorubicin. IC50 of compound 3 $(16.25 \mu \mathrm{M})$ was 1.3 times higher than that of doxorubicin (IC50 = $20.10 \mu \mathrm{M})^{19}$. Whereas, IC50 value of compound $\mathbf{1 0}$ was $9.90 \mu \mathrm{M}$ which was 2 times higher than that $(20.10 \mu \mathrm{M})$ of doxorubicin. These compounds were inactive against liver carcinoma (HEPG2), brain tumor (U251), cervix carcinoma (HELA) and breast carcinoma (MCF7) cell lines. The growth inhibitory action of the selected compounds is summarized in Table 3 and Figure 2.

Table 3: The cytotoxic activity of compounds $\mathbf{3}$ and $\mathbf{1 0}$ and Doxorubicin on Ehrlich Ascites Carcinoma cell line (EAC) and human lung carcinoma cell line (H460).

\begin{tabular}{|c|c|c|c|c|c|c|}
\hline \multirow{3}{*}{$\begin{array}{c}\text { Cpd. } \\
\text { No. }\end{array}$} & \multicolumn{5}{|c|}{ Survival fraction } & \multirow{3}{*}{$\begin{array}{c}\text { IC50 }{ }^{1} \\
(\mathrm{M} / \mathrm{mL})\end{array}$} \\
\hline & \multicolumn{5}{|c|}{ Concentration ( $\mathrm{M} / \mathrm{ml})$} & \\
\hline & 0.00 & 3.54 & 8.85 & 17.70 & 36.00 & \\
\hline & \multicolumn{6}{|c|}{ Ehrlich ascites Carcinoma ( EAC) } \\
\hline 3 & $1.00 \pm 0.11$ & $0.94 \pm 0.04$ & $0.85 \pm 0.06$ & $0.60 \pm 0.02$ & $0.50 \pm 0.01$ & $36.00 \pm 0.04$ \\
\hline 10 & $1.00 \pm 0.11$ & $0.58 \pm 0.01$ & $0.31 \pm 0.03$ & $0.15 \pm$ & $0.11 \pm 0.04$ & $5.00 \pm 0.10$ \\
\hline $\operatorname{Ref}^{2}$ & $1.00 \pm 0.11$ & $1.00 \pm 0.09$ & $0.95 \pm 0.05$ & $0.65 \pm 0.03$ & $0.42 \pm 0.01$ & $29.50 \pm 0.06$ \\
\hline & \multicolumn{6}{|c|}{$\begin{array}{l}\text { Lung carcinoma cell line }(\mathrm{H} 460) \\
\end{array}$} \\
\hline 3 & $1.00 \pm 0.11$ & $0.81 \pm 0.01$ & $0.73 \pm 0.03$ & $0.45 \pm 0.01$ & $0.42 \pm 0.00$ & $16.25 \pm 0.02$ \\
\hline 10 & $1.00 \pm 0.11$ & $0.74 \pm 0.11$ & $0.50 \pm 0.01$ & $0.44 \pm 0.01$ & $0.43 \pm 0.01$ & $9.90 \pm 0.07$ \\
\hline$\overline{\operatorname{Ref}^{2}}$ & $1.00 \pm 0.11$ & $1.00 \pm 0.05$ & $0.75 \pm 0.03$ & $0.55 \pm 0.01$ & $0.22 \pm 0.00$ & $20.10 \pm 0.06$ \\
\hline
\end{tabular}

Results represented as mean of three repeated experiments \pm SE

${ }^{1}$ IC50 $=$ Dose of compound which reduces survival of tumor cell line to $50 \%$ The tested compounds were inactive against liver carcinoma (HEPG2), brain tumor (U251), cervix carcinoma (HELA) and breast carcinoma (MCF7) cell line

${ }^{2}$ Ref $=$ Doxorubicin 


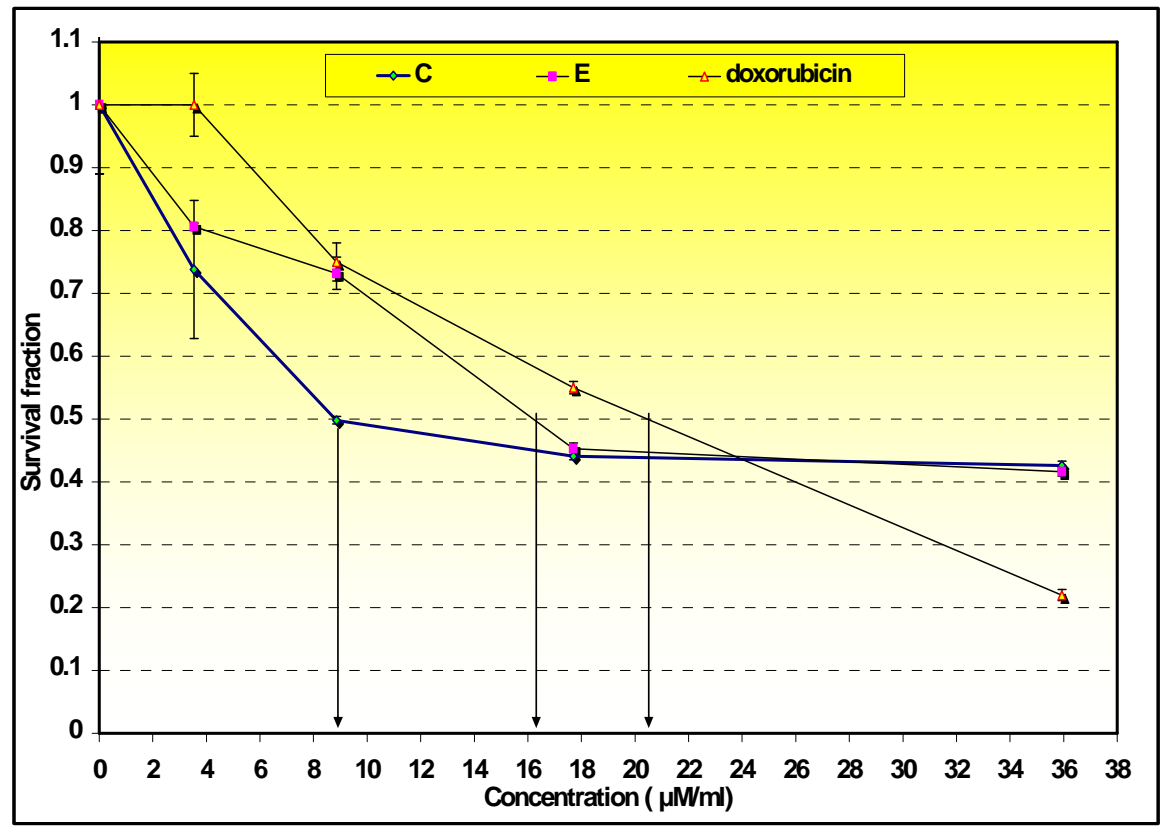

E: compound No. 3; C: compound No. 10.

Fig. 2: Cytotoxic activity of compounds $\mathbf{3}$ and $\mathbf{1 0}$ and doxorubicin on cell survival of lung carcinoma cell line (H460) arrows represented IC50 concentration.

From the study of cytotoxic activity of the tested compounds, it seems that, the introduction of an electron-withdrawing group in position 2 of coplanar tetracyclic compounds, benzo[b]phenazine derivatives is favored for activity than electron-donating group. Moreover, the parent compound, 5,12-dihydrobenzo $[b]$ phenazine-

4,11-dione was $\sim 1.3$-fold higher cytotoxic than doxorubicin, however, its classical bioisosteres, $12 \mathrm{H}-$ benzo $[b]$ phenoxazine and 12H-benzo[b]phenothiazine derivatives were considerably less active. This suggests that, the number of nitrogens is considerably important as well as the presence of electron-withdrawing group which may enable hydrogen bonding with DNA.

3-Chloro-2-(2-pyridylamino)1,4-naphthoquinone (10) having an additional nitrogen showed $\sim 2$-fold the cytotoxicity of the standard drug. Rigidification of this compounds $(\mathbf{1 2 , 1 3})$ abolished the activity. 


\section{REFERENCES}

1- I. A. Shaikh, F. Johnson and A. P. Grollman, J. Med. Chem., 29, 1329 (1986).

2- K. V. Rao and C. P. Rock, J. Heterocycl. Chem., 33, 447 (1996).

3- K. V. Rao, Cancer Chemother. Rep., Part 2, 4, 11 (1974).

4- A. Lin, B. J. Lillis, J. Med. Chem., 18, 917 (1975)

5- W. A. Denny and B. C. Bagulet, In Molecular Aspects of Anticancer Drug-DNA Interactions, $2^{\text {nd }}$ ed., Waring, M. J., Neidle, S., Eds., Macmillan: London, 1994, pp. 270-311.

6- Y. Pommier, G. Capranico, A. Orr and K. W. Kohn, Nucleic Acids Rep., 19, 5973 (1991).

7- P. Fosse, B. Rene, M. C. Le Bret, C. Paoletti and J. M. Saucier, Nucleic Acids Res., 19, 2861 (1991).

8- J. C. Wang, Annu. Rev. Biochem., 65, 635 (1996).

9- G. W. Rewcastle, W. A. Denny and B. C. Baguley, J. Med. Chem., 40, 1919 (1997).

10- Y-S. Kim, S.-Y. Park, H.-J. Lee, M.-E. Suh, D Schollmeyer and C.-O. Lee, Bioorg. Med. Chem., 11, 1709 (2003).

11- N. L. Agarwal and W. Schafer, J. Org. Chem., 45, 5144 (1980).
12- I. B. Illescas, N. Martin, J. L. Segura and C. Seoane, J. Org. Chem., 60, 5643 (1995).

13- K. V. Rao and W. P. Cullen, Antibiot. Ann., 950 (1959).

14- D. J. Brusick, Cytogenetic Assays, Aberrations and SCE techniques in Carcinogenesis and Mutagenesis testing, (J.F. Douglas Ed), Human Press Inc.: Clifton, NJ., 1984, pp. 256-76.

15- P. Skehan, R. Storeng, D. Scudiero, A. Monks, J McMahon, D. Vestica, J. T. Warren, H. Bokesch, S. Kenney and M. R. Boyd, J. Natl. Cancer Inst., 82, 1107 (1990).

16- G. W. Snedecor and W. G. Cochron, Statistical Methods' Eight Edition, Louis State University press, Ames, lowa, U. (1989).

17- J. Y. Lin and Y. C. Chen, Toxicology., 16, 120 (1978).

18- P. M. Lorusso, R. Parchment, L. Demchik, J. Knight, L. Polin, J. Dzubow, C. Behrens, B. Harrison, G. Trainor and T. H. Corbett, Investigational New Drugs, 16, 287 (1998).

19- D. Peer, Y. Dekel, D. Melikhov and R. Margalit, Cancer Research, 64, 15, 7562 (2004). 